\title{
The influence of resource overlap on bacterial reproductive success in the phyllosphere
}

\author{
Rudolf O. Schlechter ${ }^{1,2,3,6}$, Evan J. Kear ${ }^{1}$, Michał Bernach ${ }^{1,2,4,6}$, \\ Daniela M. Remus ${ }^{5}$, Mitja N. P. Remus-Emsermann ${ }^{1,2,3 *}$ \\ ${ }^{4}$ Department of Electrical and Computer Engineering, University of Canterbury, Christchurch 8011, New Zealand \\ ${ }^{5}$ Protein Science and Engineering, Callaghan Innovation, School of Biological Sciences, University of Canterbury, \\ ${ }^{6}$ Institute of Microbiology, Department of Biology, Chemistry, Pharmacy, Freie Universität Berlin, Berlin, Germany
}

${ }^{1}$ School of Biological Sciences, University of Canterbury, Christchurch 8011, New Zealand

${ }^{2}$ Biomolecular Interaction Centre, University of Canterbury, Christchurch 8011, New Zealand

${ }^{3}$ Bioprotection Research Core, University of Canterbury, Christchurch 8011, New Zealand Christchurch, New Zealand.

* Corresponding author: Mitja N.P. Remus-Emsermann, m.remus-emsermann@fu-berlin.de

\begin{abstract}
The phyllosphere is colonized by rich microbial communities, despite sparse and heterogeneously distributed resources. This resource limitation is expected to drive bacterial competition, resulting in either exclusion or coexistence based on the fitness differences and resource overlap between species. Here, we investigated the impact of competition in bacterial colonization and growth of the epiphyte Pantoea eucalypti 299R (Pe299R). To that end, pairwise competition experiments between Pe299R and diverse phyllosphere-colonizing bacteria were performed in vitro and in the Arabidopsis thaliana phyllosphere. Resource overlap was determined as the similarity in resource utilization in vitro. We found an effect of both resource overlap and phylogenetic relationships in the competition outcome between Pe299R and individual competitors in vitro. To account for bacterial individuality in the phyllosphere, we employed a single cell bioreporter to determine the number of divisions that individual cells from $\mathrm{Pe} 299 \mathrm{R}$ populations underwent when challenged with individual bacterial competitors. We observed that at the single-cell level, resource utilization similarities and phylogenetic relationships were weakly correlated with Pe299R reproductive success. We observed contrasting results for two strains (Arthrobacter sp. Leaf145 and Sphingomonas melonis Fr1) that, despite sharing either a high or low resource overlap with $P e 299 R$, both negatively affected the reproductive success of Pe299R. Interestingly, we also observed facilitative effects of Methylobacterium sp. Leaf85. This work furthers the understanding of bacterial assembly processes in heterogenous environments. Our high-resolution observations are important to build an ecological framework to predict competition outcomes in the phyllosphere and to design future bacterial biocontrol applications.
\end{abstract}

Keywords: Competition, Bacterial fitness, Leaf surface, Epiphytes, Community assembly, Single cells, Plant-microbe interactions, CUSPER

\section{Significance Statement}

For plant and consumer protection, plant microbiota manipulation has recently garnered much attention. To manipulate the plant microbiota while acknowledging its ecological context, understanding the mechanisms of microbial community assembly in the phyllosphere is essential. We explored the effect of resource overlap between competing species on the fitness of a focal species at the single-cell resolution using a bottom-up approach. Using a combination of in vitro and in planta pairwise co-inoculation experiments, we found that competition outcomes are associated with resource overlap and phylogenetic relationships. Although this effect is not strong enough to promote competitive exclusion, we provide examples of competitors that affect the growth of a focal species depending on their resource overlap.

\section{Introduction}

For bacteria, the phyllosphere -mostly represented by leaf surfaces- constitutes a challenging environment where resources are limited and heterogeneously distributed $(1,2)$. This fragmented microbial habitat promotes cell-to-cell contact and should directly impact on community dynamics by short-distance interactions $(1$, $3-5)$, leading to the local competition for resources and spatial structuring of bacterial communities (6-8). Resource competition results in either exclusion or coexistence depending on the strength of interspecies interactions in relation to conspecific interactions, that is, interactions within a species (9). Coexistence maintains community diversity and is promoted when competition against a different species is weaker than competing with conspecifics. The strength of interspecific competition can be alleviated by mechanisms that increase niche differences, such as niche overlap (10). 

available under aCC-BY-NC-ND 4.0 International license.

Schlechter et al., 20 JAN 2022 - preprint - BioRxiv

Due to the environmental heterogeneity of the phyllosphere, 'coarse-grained' investigations, such as those considering only a whole leaf or plant as unit of investigation, are of limited suitability when studying local interactions of leaf-associated bacteria. Therefore, to better understand bacterial growth dynamics in the phyllosphere, individual cells must be the subject of investigation, as every cell may experience a different fate (5). A bioreporter used to estimate the number of divisions of individual cells, CUSPER ("repsuc" read backwards, from "reproductive success"), has shown that bacteria experience high variations of reproductive success in the phyllosphere $(11,12)$. Due to the heterogeneously distributed and limited resources on leaves, the reproductive success of a bacterial cell depends on the local habitability. Consequently, it has been shown that leaves that were pre-colonized with a conspecific strain reduced the reproductive success of CUSPER bioreporter cells proportional to the pre-colonizer density (13). However, interspecific resource competition at the singlecell resolution in the phyllosphere has not been explored.

Here, we tested if resource competition in vitro correlates with reproductive success in planta. We expect that strong negative impacts in vitro will also strongly reduce single cell reproductive success in the phyllosphere. To that end, the epiphyte Pantoea eucalypti 299R (Pe299R) was challenged with a range of diverse phyllosphere-colonizing bacteria in pairwise competition experiments. Similarities in resource utilization profiles in vitro were used as a proxy to estimate resource overlap and to select potential strong and weak competitors of Pe299R. Finally, bacterial fitness in the phyllosphere was evaluated using the CUSPER bioreporter in the presence of competitors with different degrees of resource overlap to Pe299R.

\section{Results}

Bacterial Resource Utilization Profiles. A resource utilization profile for a group of phyllosphere-associated bacterial strains (Table 1, Appendix SI, Table S1) was determined by growing each strain in defined minimal media (MM) supplemented with either $0.2 \% \mathrm{w} / \mathrm{v}$ glucose, fructose, malate, sorbitol, or methanol, and comparing the biomass yields across these different growth conditions. Carbon sources were selected based on the detection and abundance of metabolites extracted from whole arabidopsis leaves (14). Additionally, methanol was chosen as it is released
Table 1. Strains used in this study.

\begin{tabular}{rlc}
\hline Genus species & Strain & Abbreviation \\
\hline Acidovorax sp. & Leaf84 & AcidoL84 \\
Aeromicrobium sp. & Leaf245 & AeromL245 \\
Agreia sp. & Leaf335 & AgreiL335 \\
Arthrobacter sp. & Leaf145 & ArthrL145 \\
Bradyrhizobium sp. & Leaf396 & BradyL396 \\
Methylobacterium sp. & Leaf85 & MethyL85 \\
Methylorubrum sp. & Leaf92 & MethyL92 \\
Microbacterium sp. & Leaf320 & MicroL320 \\
Pseudomonas koreensis & P19E3 & PkP19E3 \\
Pseudomonas syringae & B728a & PssB728a \\
Rhodococcus sp. & Leaf225 & RhodoL225 \\
Sphingomonas melonis & FR1 & SmFR1 \\
Sphingomonas phyllosphaerae & FA2 & SpFA2 \\
Sphingomonas sp. & Leaf17 & SphinL17 \\
Sphingomonas sp. & Leaf357 & SphinL357 \\
Pantoea eucalypti & 299R & Pe299R \\
\hline
\end{tabular}

from leaves $(15,16)$. Hierarchical clustering showed four clades based on the growth of the studied strains in the amended minimal medium (Fig. 1A). The focal species Pe299R grew on glucose, fructose, and malate, but not on either sorbitol or methanol. The clustering grouped Pe299R closely with the gammaproteobacterium Pseudomonas koreensis P19E3 (PkP19E3) and the actinobacterium Arthrobacter sp. Leaf145 (ArthrL145) which both showed similar growth in glucose, fructose, and malate. To a lesser extent, Pe299R grouped with members of Alpha-, Beta-, and other Gammaproteobacteria, as well as the actinobacterium Aeromicrobium sp. Leaf245 (AeromL245). The alphaproteobacterium Methylobacterium sp. Leaf85 (MethyL85), Methylorubrum sp. Leaf92 (MethyL92), and Bradyrhizobium sp. Leaf396 (BradyL396) grew most efficiently in malate compared to the other resources. A fourth clade was defined by strains with the lowest overall biomass yield in the tested growth conditions, including Sphingomonas sp. Leaf357 (SphinL357) and Rhodococcus sp. Leaf225 (RhodoL225) with a comparatively lower growth than a

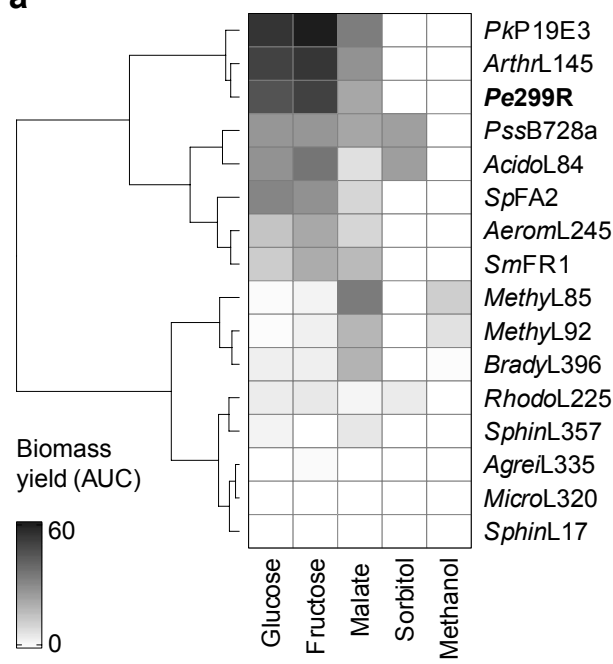

b

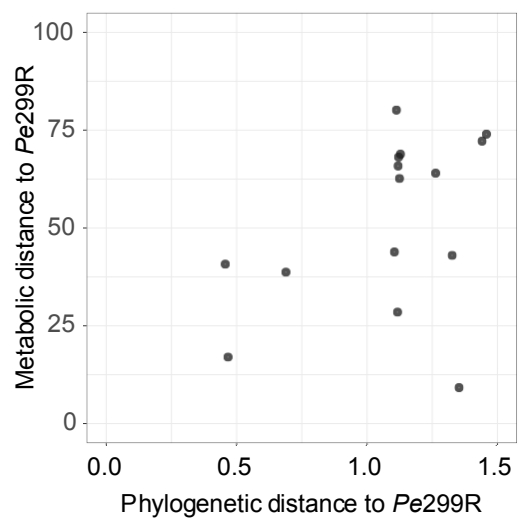

Figure 1. Resource utilization profile of phyllosphere-associated bacteria. $(A)$ Species resource utilization matrix. Biomass yield is depicted as the area under the growth curve (AUC) from $\mathrm{OD}_{600}$ measurements. Focal species Pe299R is highlighted in bold. Hierarchical clustering was based on similarities in resource utilization profiles between species. (B) Relationship between phylogenetic distance (substitutions per site) and metabolic distance (Euclidean distance from $A$ ) of potential competitor species and Pantoea eucalypti 299R (Pe299R). Refer to Table 1 for the full name of each phyllosphere-associated strain. 
strains in the other clades for glucose, fructose, malate, and sorbitol, while Agreia sp. Leaf335 (AgreiL335), Microbacterium sp. Leaf320 (MicroL320), and Sphingomonas sp. Leaf17 (SphinL17) failed to grow in every growth condition. To test whether metabolic distance correlates with phylogenetic distance between the tested strains and Pe299R, metabolic distances were calculated as the Euclidean distances between the resource profiles of $P$ e299R and a second species. Phylogenetic distances were calculated as the distance between branches in a reconstructed phylogenetic tree (Appendix SI, Fig. S1). A weak correlation was found between phylogenetic and metabolic distance (Fig. 1B, $p$-value $=0.123$, adjusted $R^{2}=0.109$, Pearson's correlation $=0.416$ ), suggesting that phylogeny does not explain metabolic differences between Pe299R and the rest of the selected phyllosphere-associated bacteria.

Resource Competition In Vitro. The ability of a competitor to affect the growth of a fluorescently red-labeled Pe299R (Pe299R::mSc, Appendix SI, Table S1) was evaluated in vitro. The growth of each strain was measured in MM supplemented with multiple resources $\left(\mathrm{MM}_{5 \times \mathrm{c}}, 0.125 \% \mathrm{w} / \mathrm{v}\right.$ of a mix of glucose, fructose, malate, sorbitol, and methanol) to compare growth yields of potential competitors of Pe299R::mSc. The resource concentration was chosen based on the highest fluorescence readout without compromising growth of $P e 299 \mathrm{R}$ at the lower range of resource concentration (Appendix SI, Fig. S2). In general, most competitor bacteria were able to grow on $\mathrm{MM}_{5 \times \mathrm{C}}$, except for $\mathrm{Mi}$ croL320 (Fig. 2, Appendix SI, Fig. S3). Strains that yielded growth comparable with Pe299R were PkP19E3, ArthrL145, Pseudomonas syringae pv. syring ae B728a (PssB728a), Sphingomonas phyllosphaerae FA2 (SpFA2), and Acidovorax sp. Leaf84 (AcidoL84).

At least two strains of each clade from the resource utilization profile that also grew on the $M_{5 \times c}$ growth condition were selected to test their competitiveness against Pe299R in vitro. To test the effect of a strain on Pe299R growth, Pe299R::mSc was mixed in equal ratios with a single phyllosphere-associated strain and fluorescence intensity was measured over time as a proxy for Pe299R::mSc biomass (17). Then, Pe299R::mSc growth yields in competition with an interspecific competitor was normalized by the effect of Pe299R::mSc in intraspecific competition (i.e., Pe299R::mSc mixed with its non-tagged parental strain). In these conditions, the presence of PkP19E3, PssB728a, or ArthrL145 negatively impacted the growth of Pe299R::mSc, while the other tested strains exhibited a lower impact on the growth of Pe299R::mSc (Fig. 3A). Based on the maximal fluorescence intensity of the Pe299R::mSc as monoculture, the presence of other strains did not lead to increased growth of Pe299R::mSc, suggesting that all strains were true competitors. Additionally, no competitor exhibited direct inhibitory effects on Pe299R (SI Appendix, Fig. S4), suggesting that resource competition rather than interference competition (e.g., antibiosis) is driving species interactions between the tested bacteria and Pe299R.

To determine the relationship between resource overlap, phylogenetic relationships, and competitiveness in vitro, a metabolic distance was estimated based on the similarity of resource utilization profile between competitors and Pe299R::mSc. Both metabolic and phylogenetic distance between a competitor and Pe299R::mSc were used as explanatory variables to evaluate their effect on the ability to affect Pe299R::mSc growth. Regression analysis found an interaction between metabolic and phylogenetic

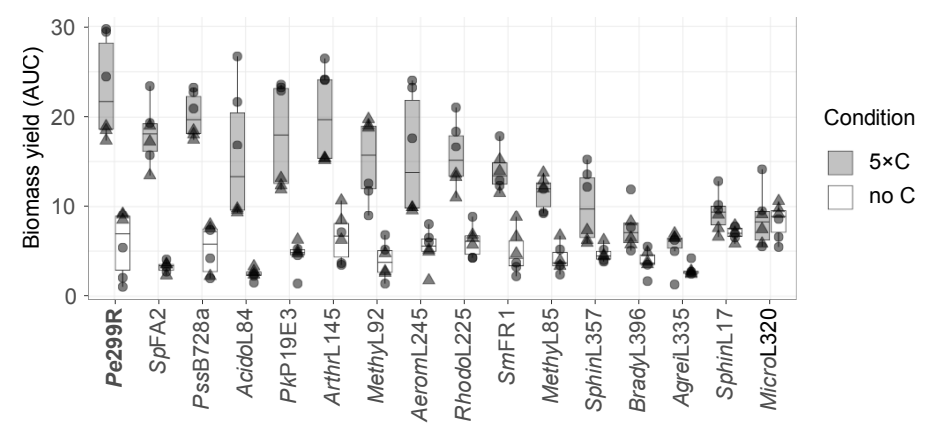

Figure 2. Growth of phyllosphere-associated bacteria in multiple carbon sources. Growth of phyllosphere-associated bacteria in minimal media supplemented with $0.125 \% \mathrm{w} / \mathrm{v}$ of mixed carbon sources $\left(\mathrm{MM}_{5 \times \mathrm{C}}\right)$. Focal species Pe299R is highlighted in bold. Biomass yield was determined as the area under the growth curve (AUC) from $\mathrm{OD}_{600}$ measurements of individual species grown in a mix of five carbon sources ( $5 \times \mathrm{C}$ : glucose, fructose, malate, sorbitol, methanol). As negative control, MM without carbon sources was used. Different shapes indicate results from independent experiments.

distance (Figure $3 B, p$-value interaction $<0.05$, adjusted $R^{2}=0.80$ ), suggesting that the response of a competitor on Pe299R growth depends on both metabolic and phylogenetic differences. In summary, both resource overlap and phylogenetic relatedness have a role in the competitiveness of a bacterial strain in affecting Pe299R::mSc fitness in vitro.

Pe299R Single-Cell Reproductive Success in Competition. Here, an improved version of the CUSPER bioreporter plasmid was constructed (SI Appendix, Fig. S5) and was used to develop Pe299R::Tn7::GmR ::mSc (pProbe_CUSPER) -hereafter referred to as Pe299R CUSPER $^{-}$. Pe299R PUSPER $_{\text {is }}$ a bioreporter that estimates the reproductive success (RS) of immigrant cells in a new environment by back-calculating the number of divisions a cell underwent since its arrival (11-13). Reproductive success was determined by measuring the decrease in single cell fluorescence compared to the mean fluorescence of the population at time zero $\left(t_{0}\right)$. Under optimal homogeneous conditions, it is expected that the entire population will divide until the lower limit of detection for the GFP signal is reached. By contrast, factors such as environmental heterogeneity and presence of competitors are expected to decrease the success of the immigrant population. The newly developed Pe299R

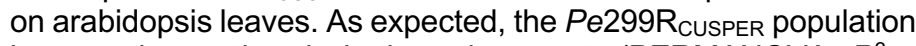
increased over time in both environments (PERMANOVA, $R^{2}=$ $67.5 \%, p$-value $=0.001)$. In liquid culture, the cell population divided at regular intervals $(\sim 1 \mathrm{~h})$, suggesting that the reproductive success of individual cells within a population was normally distributed (SI Appendix, Figure S6A). By contrast, on arabidopsis leaves, subpopulations differentiated from each other over time, resulting in a non-normally distributed $P$ e299R RUSPER $_{\text {cell popula- }}$ tion after $36 \mathrm{~h}$ ( $S$ I Appendix, Fig. S6B). In both cases, the limit of detection was approximately five divisions before the fluorescence of cells was not significantly different from the background ( $S I A p$ pendix, Fig. S7).

The effect of a competitor on the reproductive success of Pe299R CUSPER was evaluated in the arabidopsis phyllosphere. A set of seven phyllosphere-associated bacteria: PkP19E3, PssB728a, ArthrL145, Pe299R, MethyL85, SmFR1, and RhodoL225, were selected based on their ability to affect $P$ e299R 
a

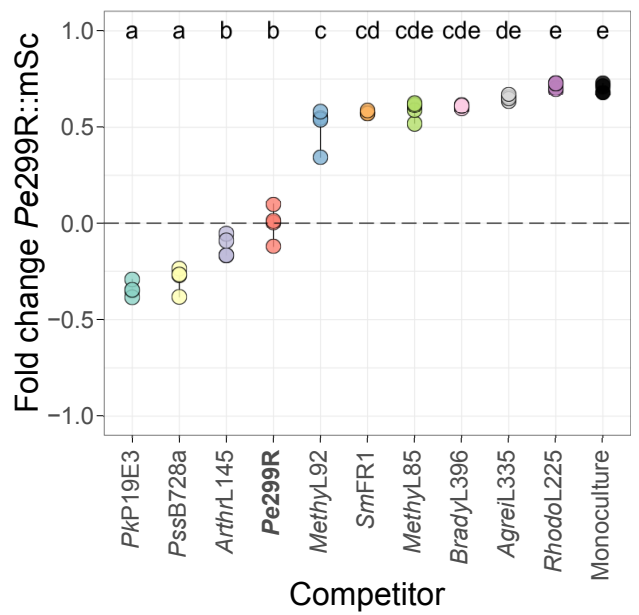

b

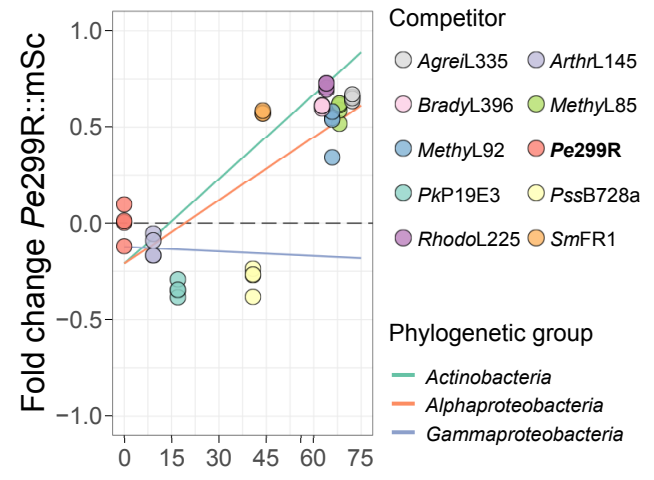

Metabolic distance relative to $P$ e299R

Figure 3. Fitness of $P$. eucalypti 299R in competition with phyllosphere-associated bacteria in liquid culture. $(A)$ Ranked fold change in fluorescence of $P$. eucalypti 299R::Tn7::mSc::Gm ${ }^{R}(P e 299 R:: m S c)$ in interspecific competition relative to intraspecific competition $(P e 299 R$, bold) in MM $5 \times c$. Differences between groups were tested with ANOVA $(\alpha=0.05)$ and letters display multiple pairwise comparisons using Tukey's post hoc test with Bonferroni correction. (B) Relationship between metabolic distance between Pe299R::mSc and a second species on Pe299R fitness. Continuous colored line indicates fitted model at different degrees of phylogenetic relatedness between a competitor (green: Actinobacteria, orange: Alphaproteobacteria, purple: Gammaproteobacteria) and Pe299R::mSc (Gammaproteobacteria), based on the regression model $y=-0.12-0.057 X_{\text {phylogeny }}-0.00755 \mathrm{X}_{\text {metabolism }}+0.016094 \mathrm{X}_{\text {phylogeny }}$ $\mathrm{X}_{\text {metabolism. }} \operatorname{In}(A)$ and $(B)$, a horizontal dashed line indicates no fold change $(\mathrm{y}=0)$ with respect to intraspecific competition as a reference.

growth in vitro. Four-week-old arabidopsis plants were co-inoculated with $P$ e299R estimate single-cell reproductive success in planta as well as the changes in population densities. As expected, we first observed that in all conditions, Pe299R CUSPER $_{\text {differentiated into subpopula- }}$ tions over time with different degrees of reproductive success (Fig. 4). Pe299R CUSPER cells have divided with a median between four to five times at 24 and $36 \mathrm{~h}$ ( median $\left._{R S}=3.98-5.11\right)$. However, the presence of competitor species influenced the distribution of Pe299R ${ }_{\text {CUSPER }}$ populations during leaf colonization (Kruskal-Wallis, $p<0.0001$ ). Particularly, the presence of the competitors ArthrL145, PssB728a, RhodoL225, and SmFR1 decreased the reproductive success of Pe299R Cusper at $24 \mathrm{~h}$ (Fig. 4). This effect was maintained over time when ArthrL145 or SmFR1 was present, but only transient in the presence of RhodoL225, as no differences were observed at $36 \mathrm{~h}$ for this competitor (Fig. 4). By contrast, PkP19E3, PssB728a, and MethyL85 showed to have a positive effect on the reproductive success of Pe299R 4). We also observed that when Pe299R or ArthrL145 were competitors, the median RS of Pe299R ${ }_{\text {CUSPER }}$ decreased from 24 to 36 $\mathrm{h}$ (Dunn's test, $p<0.05)$. The generalized linear mixed model with the best fit $\left(S I\right.$ Appendix, Table S2, pseudo- $R^{2}=0.043$ ) showed that the observed reproductive success in the realized

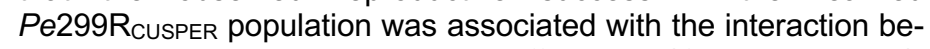
tween metabolic and phylogenetic differences (GLMM, $p<0.05$ ), and the interaction between metabolic differences and time of sampling (GLMM, $p=0.016$ ). These results suggest a significant but rather small effect of a competitor species in the reproductive success of $P$ e299R based on their phylogenetic relationships and resource overlaps in the phyllosphere.

We found that the presence of a competitor also affected Pe299R CUSPER over time at the population-level (two-way ANOVA, $F=2.76, p=0.0025$ for the interaction between the presence of competitor and time of sampling). However, single-cell and population-level estimations of the fitness effect of Pe299R CUSPER in competition in the phyllosphere were partially consistent. For example, only the presence of SmFR1 negatively affected the density of the whole Pe299R and ArthrL145 positively affected the focal strain at $36 \mathrm{~h}$ (Fig. 5). The effect of other strains was not statistically different to the pop-

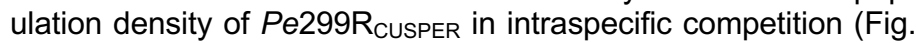
5). Multiple regression analysis suggested that population-level density of $P$ e299R in competition is associated with an interaction between metabolic differences an phylogenetic relationships with its competitors, as well as the time of sampling (Linear mixed-effects model, $\beta_{\text {interaction }}=-0.031$, marginal $R^{2}=0.54$, conditional $R^{2}$ $=0.92, p=0.0008$ ).

The distribution of immigrant cells that experienced different levels of reproductive success was analyzed as relative fractions of the initial population. Subpopulations were assigned based on the estimated reproductive success of an arrival cell, that is, the number of cells that divided from 0 to $>5$ times after inoculation (SI Appendix, Table S3). The fraction of each Pe299R was then used to compare the differences in distribution of Pe299R We found that the sampling time explained most of the variation in the composition of the Pe299R $R^{2}=74 \%, p=0.001$ ) rather that the presence of a competitor (PERMANOVA, $R^{2}=2.3 \%, p=0.217$ ). However, we noticed that the subpopulation of Pe299R CUSPER cells that divided five times was larger and contributed mostly to the final population when PkP19E3, PssB728a, and MethyL85 were present at $36 \mathrm{~h}$, in com-

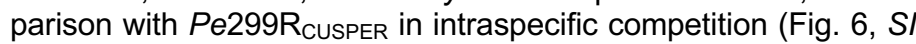
Appendix, Fig. S8). However, these observations were not linked to either phylogeny (linear regression, $p=0.69$ ) or the metabolic differences estimated in vitro (linear regression, $p=0.99$ ). 
bioRxiv preprint doi: https://doi.org/10.1101/2022.01.20.477054; this version posted January 21, 2022. The copyright holder for this preprint (which was not certified by peer review) is the author/funder, who has granted bioRxiv a license to display the preprint in perpetuity. It is made available under aCC-BY-NC-ND 4.0 International license.

Schlechter et al., 20 JAN 2022 - preprint - BioRxiv

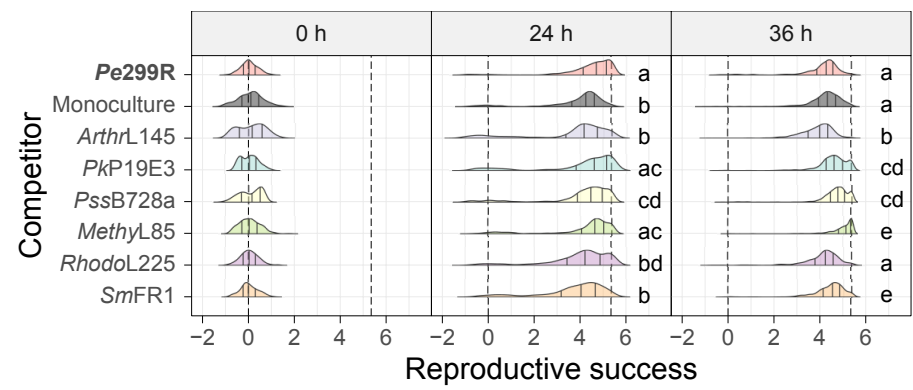

Figure 4. Single-cell reproductive success of Pe299R tions in competition in the phyllosphere. Distribution of the reproductive

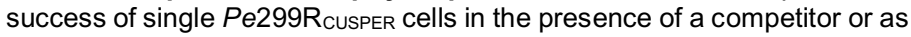
monoculture at 0,24 , and $36 \mathrm{~h}$ post-inoculation onto arabidopsis leaves. Intraspecific competition (Pe299R) is highlighted in bold. Each violin plot indicates the median and the interquartile range. Groups within each time point were compared using Kruskal-Wallis $H$ test by ranks, with a significance level of $\alpha=0.0001$. Letters display multiple pairwise comparisons between groups within each time point using Dunnett's tests with Bonferroni correction.

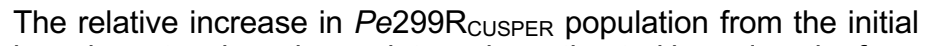
inoculum at a given time point can be estimated based on the fraction of cells in a particular subpopulation and the number of divisions that a cell is expected to undergo upon arrival in the phyllosphere (12). This increase estimated by single-cell measurements was highly correlated with the increase in population size at the CFU level (SI Appendix, Fig. S9, $R^{2}=0.79$ ). However, the increase in Pe299R ated with the sampling time (two-way ANOVA, $p<0.05$ ) but not the presence of a competitor (two-way ANOVA, $p=0.36$ ) ( $S I A p$ pendix, Fig. S10). This pattern was not explained by the phylogenetic relationships (linear regression, $p=0.48$ ) or the metabolic differences estimated in vitro (linear regression, $p=0.61$ ) between Pe299R and a competitor, but only by sampling time (linear regression, $p=0.023$ ). Our findings suggest that competition in vitro and resource overlap are not the best predictors of bacterial population increase in the phyllosphere.

\section{Discussion}

In this study, the relationship between resource competition and reproductive success in the phyllosphere was investigated. The competitive ability of a taxonomically diverse group of phyllosphere-associated bacterial species were estimated in vitro and used to rank their competitiveness against Pe299R in both liquid culture and the arabidopsis phyllosphere. Pe299R was selected as a focal species as it is a model organism that has been widely used to understand bacterial physiology and ecology in the phyllosphere $(2,7,18-21)$. However, its interactions with other species in this environment has been underexplored. Lindow and Wilson (1994) showed a negative correlation between coexistence and similarity in resource utilization for a number of pairs of phyllosphere-associated bacteria, including Pe299R (22). Here, a wide range of bacterial species, including the understudied Gram-positive phyllosphere-associated bacterial phylum Actinobacteria, were included to understand whether similarity in resource use had a phylogenetic signal. Our results show that the clustering of species based on their resource utilization profiles did not correlate with their phylogenetic relationships. Particularly, Pe299R grouped closely with the gammaproteobacterium PkP19E3 and the actinobacterium ArthrL145. This is in line with the findings that carbon utilization is a trait that is widespread and not phylogenetically conserved in Bacteria (23).

Potential competitors were selected according to their similarity in resource use with $P$ e299R, as competitive exclusion is expected to be favored under high metabolic overlap in conditions where resources are shared (24). Among these competitors, PkP19E3 and ArthrL145 were of special interest due to their high metabolic similarity, but different degrees of phylogenetic relationships with $P$ 2299R. Other groups included representative members of the phyllosphere microbiota, such as the highly abundant Sphingomonas, Methylobacterium, and Pseudomonas spp. (25), and less abundant members, such as Bradyrhizobium, Agreia, and Rhodococcus spp. (26). The impact of competitor species on the fitness of Pe299R in vitro was most likely a consequence of resource competition, as no direct antagonistic effects were found for the tested bacteria on Pe299R in a double layer assay (SI Appendix,

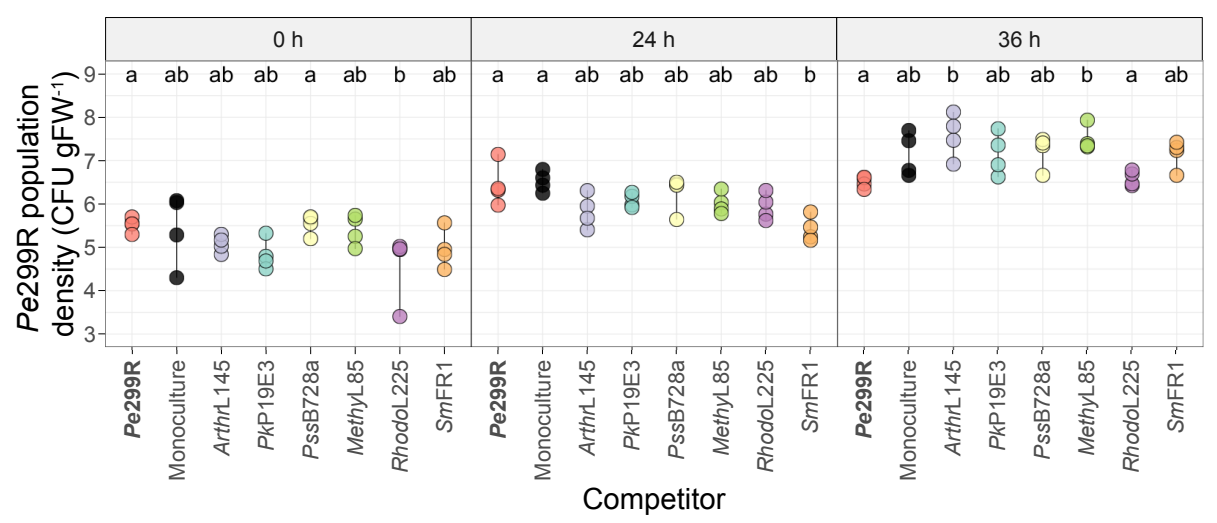

Figure 5. $P$. eucalypti $299 \mathrm{R}$ population density in competition in the phyllosphere. Population size of $P$. eucalypti $299 \mathrm{R}:: T n 7:: \mathrm{mSc}:: \mathrm{Gm}{ }^{\mathrm{R}}$ (pProbe_CUSPER) (Pe299R_usper) on arabidopsis plants at 0, 24, and $36 \mathrm{~h}$. Pe299Rcusper was co-inoculated with a near-isogenic strain Pe299R, or with a different phyllosphere-associated bacteria in equal ratios. Pe299R $(P e 299 R)$ is highlighted in bold. Each data point represents the CFU of $P$ e299R per gram of fresh leaf weight $\left(C F U \mathrm{gFW}^{-1}\right)$ of independent plants $(\mathrm{n}=$ 4). Groups were compared using two-way ANOVA, with a significance level of $\alpha=0.05$. Letters display multiple pairwise comparisons between groups within each time point using Tukey's post hoc test with Bonferroni correction. 


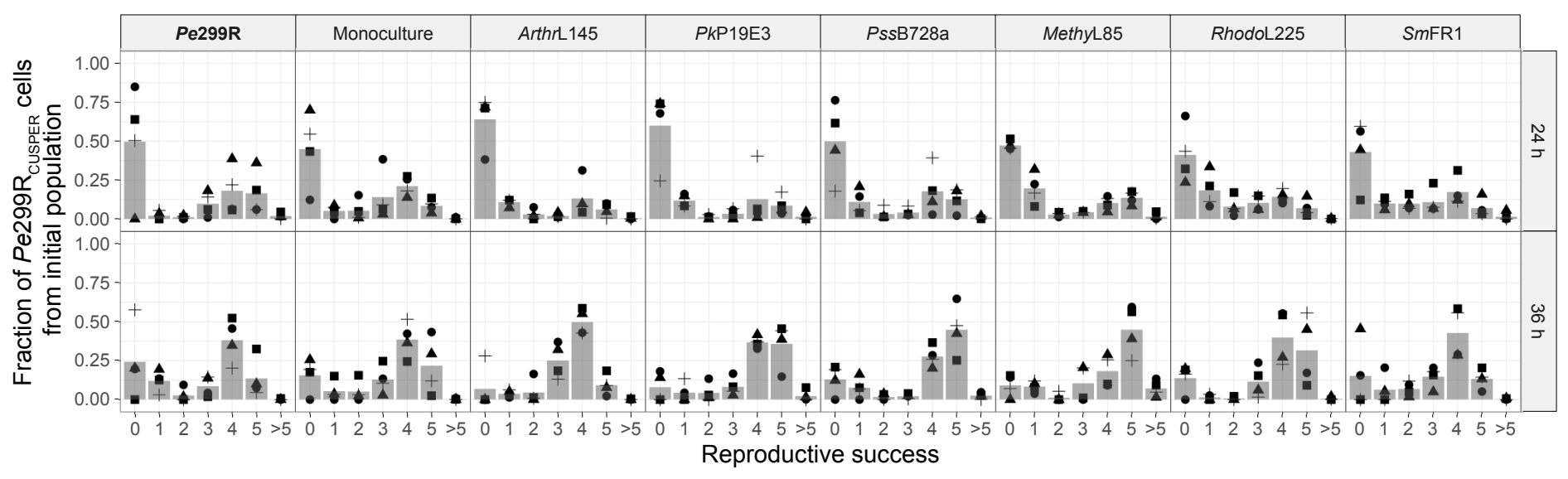

Figure 6. Relative fractions of Pe299R in presence of different competitors. Each data point represents a fraction of the subpopulations per independent biological replicate (symbols) and the bar represents the mean relative fraction for all replicates. Intraspecific competition (Pe299R) is highlighted in bold.

Fig. S4). This is not surprising since interference competition through antibiosis is relatively rare between phyllosphere-associated bacteria, as fewer than $2 \%$ of binary interactions resulted in growth inhibition (27). Furthermore, inhibitions are mostly driven by only two bacterial orders, the Pseudomonadales and Bacillales, and the only candidates belonging to Pseudomonadales (PkP19E3 and PssB728a) did not inhibit Pe299R.

The epiphytes PkP19E3 and ArthrL145, as well as PssB728a, showed a strong negative effect on Pe299R fitness in vitro. In contrast, competitors with low resource similarity (e.g., SmFR1, MethyL85, MethyL92, BradyL396, AgreiL335, and RhodoL225) had a minor impact on Pe299R fitness, suggesting a strong relation between metabolic similarities and Pe299R fitness in vitro. A regression model showed an interaction effect of phylogenetic and metabolic differences on the fitness of Pe299R when in competition for multiple resources. These results suggest that under high resource overlap, phylogeny has a small effect on the growth of Pe299R. However, at lower resource overlap, distantly related species had a smaller impact on Pe299R fitness. A recent study on the interactions between and within Pseudomonadales and Enterobacterales showed that interactions between species have both a phylogenetic and metabolic signal in vitro (28), where negative interactions are more common between closely-related species, which also happened to have a higher level of resource overlap. Overall, the findings of the in vitro observations indicate that similarities in resource usage and phylogenetic relationships explain the strength of pairwise competitive interactions.

The redeveloped bioreporter Pe299R CUSPER $_{\text {was }}$ used to estimate the single-cell reproductive success of $P$ e299R in competition with selected phyllosphere-associated strains in planta, which were shown to affect Pe299R fitness in vitro to varying degrees. This bioreporter relies on the fluorescence intensity of individual cells, which can be traced back to estimate the number of divisions a cell underwent based on the dilution of a fluorescent protein after cell division (11). This bioreporter was instrumental in that bacterial populations in the phyllosphere separate into subpopulations over time (11). In contrast to the initial CUSPER bioreporter, the redeveloped bioreporter produces an additional red fluorescent protein and carries a recently developed green fluorescent protein $\mathrm{mClo-}$ ver3 on a plasmid, rather than a single copy of GFPmut3 that was chromosomally inserted. As expected, the results using the redeveloped and enhanced Pe299R CUSPER $_{\text {were }}$ consistent with the first iteration: growth of $P e 299 \mathrm{R}$ in liquid culture was normally distributed, while growth in the arabidopsis phyllosphere resulted in subpopulations that experienced different numbers of divisions ( $S I$ Appendix, Fig. S6 and S7). These results suggest that the variable habitability of the phyllosphere is a common feature of plant species, as previous works on CUSPER were performed on bush bean leaves (Phaseolus vulgaris). The differences in reproductive success of Pe299R subpopulations can be linked to heterogeneous amounts of resources such as carbohydrates and water that immigrant bacterial cells are exposed to upon arrival on the leaf surface $(1,2,29)$. After 36 hours, we observed a reduction of cells that did not divide after arriving in the phyllosphere $(R S=0)$ (Fig. 6, SI Appendix Fig. S6B and S8). This is likely the result of dying cells, rather than initially unsuccessful cells migrating to new sites that would allow them to grow, as bacterial relocation has been suggested to happen during early stages of colonization in the phyllosphere (20). Furthermore, there were no signs of dew in our system that was under controlled relative humidity conditions that would allow for migration to happen. However, a fraction of cells with $\mathrm{RS}=0$ is expected to be true unsuccessful cells that could have undergone cell death and lysed, which cannot be determined with our bioreporter.

Recognizing the variable fate of bacterial cells during leaf colonization, we tested the effect of resource overlap in competitive bacterial interactions at the single-cell level. Particularly, we found that ArthrL145 significantly affected the growth of Pe299R by restricting the reproductive success of $P e 299 \mathrm{R}$ to a median of four cell divisions. Arthr 145 was selected as a strong competitor of Pe299R as this strain showed the highest similarity in resource usage, biomass yield in multiple carbon source growth condition, and a negative impact on Pe299R fitness in vitro. Altogether, ArthrL145 was the best example of resource overlap being a stronger predictor of competition outcome than phylogeny. Only recently, ArthrL145 was also shown to have a protective effect in arabidopsis against the foliar pathogen Pseudomonas syringae 
pv. tomato DC3000 (PstDC3000) through a mechanism independent of the BAK1/BKK1-associated plant immune response (30), suggesting that microbe-microbe interactions such as resource overlap, could be driving the reduction in Pe299R populations as well.

PkP19E3 and PssB728a were also selected as a strong competitor because of its similarity in resource usage and phylogenetic relationships, and because they had a strong negative effect on Pe299R in competition in vitro. Unexpectedly, these strains positively affected the growth of more successful Pe299R subpopulations, showing an increase in relative abundance of cells that divided five times or more (Fig. 6). This suggests that mechanisms other than resource competition were influencing the interactions between Pe299R and PkP19E3 or PssB728a. Pseudomonas spp. are recognised for producing biosurfactants, which are amphiphilic compounds that improve the permeability of resources onto the leaf surface and increase bacterial survival due to their water retaining hygroscopic nature (31-33). By modifying their local environment, $P$ seudomonas spp. could indirectly benefit $P e 299 R$. Alternatively, these strains could engage in cooperative interactions such as cross-feeding, as it was recently shown that potential between Pantoea sp. and Pseudomonas koreensis in the Flaveria robusta leaf apoplast (34). However, further investigations are required to understand the mechanisms that result in beneficial interactions in the phyllosphere.

We tested whether competition can be influenced by resource overlap among phyllosphere-associated bacteria. While high resource overlap was expected to have a negative impact on Pe299R growth, we also expected that low resource overlap would result in neutral or positive effects on Pe299R. Indeed, we found that the presence of MethyL85 consistently supported Pe299R growth in the phyllosphere, both at the single-cell and CFU level. MethyL85 belongs to a group of resource specialists and facultative methylotrophs from the genus Methylobacterium. Methanol utilization is a fitness advantage in the phyllosphere, as methylotrophs can utilize the released methanol from the plant cell wall metabolism (35). As one carbon metabolism is highly overrepresented in proteomes of methylobacteria on leaves $(25$, 36), it is expected that MethyL85 utilizes methanol as a carbon source and does not compete with Pe299R for their preferred resources. Possibly, additional biomass and surfactant production may act as a water retaining factor which increases survival and spread of bacteria $(31,32,37)$.

In other cases, strains such as SmFR1 showed a transient negative effect on the growth rate of Pe299R subpopulations in the phyllosphere, despite displaying a low resource usage similarity and low impact on Pe299R fitness in vitro. The poor relationship between these factors and the ability of some strains to affect Pe299R reproductive success could be due to indirect effects, such as through modulation of the environment via host-microbe interactions. SmFR1 has been previously shown to decrease the population density of PstDC3000 in arabidopsis, potentially through priming of plant immune defense responses $(30,38,39)$. However, the spatio-temporal dynamics of these changes and their significance to other microbial inhabitants remain elusive.

Overall, we observed a relationship between the similarity in resource usage between pairs of species and single-cell reproductive success. It has been shown that similarity in resource usage is negatively correlated with the level of coexistence between pairs of epiphytic bacteria $(22,40)$. Our findings support the hypothesis that resource competition plays a role in community assembly processes in the phyllosphere. The observed relationship suggests that the similarity in resource usage profiles impact to some extent on the reproductive success of a population in interspecific competition compared to intraspecific competition. This relationship was stronger and more predictive in a homogeneous environment than in the phyllosphere. These differences resulted in a relative increase of cells that were less successful when competitors with similar resource profiles were present. However, competition did not drive the exclusion of any species in any of the tested pairwise interactions, suggesting that the strength of competition is alleviated by either fitness differences or stabilizing mechanisms, enabling the coexistence of the studied species in the phyllosphere. These findings establish a link between resource overlap and reproductive success, supporting the notion that resource competition is one of many factors that impacts on community assembly processes in a spatial and temporal manner in heterogeneous environments such as the phyllosphere.

Understanding the impact of resource competition in bacterial community assemblage in the phyllosphere has major implications in developing effective biocontrol strategies against biotic stresses. Many bacterial foliar pathogens undergo an epiphytic lifestyle that is characterized by population growth before colonizing the plant host endophytic compartments (41). The rational design of biocontrol agents or communities that effectively reduce pathogen populations in the phyllosphere through competitive interactions with native phyllosphere-associated microbial residents could prevent crop losses caused by microbial diseases.

\section{Materials and Methods}

Bacterial Strains and Growth Conditions. Pe299R and representative phyllosphere-associated bacterial strains are listed in Table 1 (Appendix SI, Table S1). Pe299R was used to construct the constitutively red-fluorescent protein producing strain Pe299R::mSc and the CUSPER bioreporter strain Pe299R CUSPER (Appendix SI, Materials and Methods). Bacteria were routinely grown in Reasoner's $2 \mathrm{a}$ agar or broth (R2A, HiMedia) at $30^{\circ} \mathrm{C}$. Minimal media was used to evaluate growth and competition for defined carbon sources, as described in (17). Carbon sources used were glucose, fructose, sorbitol, malate, and methanol.

Carbon Utilization Profiles. Each strain was grown at $30^{\circ} \mathrm{C}$ in R2A broth until the late stationary phase. Cells were then harvested by centrifugation at $2,000 \times g$ for $5 \mathrm{~min}$, washed twice in PBS, and resuspended in MM to an optical density $\left(\mathrm{OD}_{600}\right)$ of 0.5 . Afterwards, $20 \mu \mathrm{L}$ of bacterial suspension were added to $180 \mu \mathrm{L}$ of $\mathrm{MM}$ supplemented with $0.2 \% \mathrm{w} / \mathrm{v}$, or $0.2 \% \mathrm{v} / \mathrm{v}$ for methanol, of a carbon source in flat bottom 96-well microtiter plates (Costar) with four technical replicates per condition. Minimal medium without added carbon source was used as a negative control. Optical density was measured in a FLUOstar Omega microplate reader (BMG Labtech) for 5 days in $24 \mathrm{~h}$ intervals. The experiments were conducted twice independently.

Competition for Carbon Sources. Competition assays were performed in MM supplemented with a mix of glucose, fructose, sorbitol, malate, and methanol $\left(\mathrm{MM}_{5 \times \mathrm{C}}\right)$. Suitable carbon source concentrations were determined as explained in Appendix SI, Material and Methods. Pe299R::mSc was competed against individual non-fluorescent competitors by mixing both strains in a $1: 1 \mathrm{OD}_{600}$ ratio, as described elsewhere (17). Briefly, flat bottom 
96-well microtiter plates (Costar) were seeded with $200 \mu \mathrm{L} \mathrm{MM}_{5 \times \mathrm{C}}$ and a defined mixed bacterial suspension $\left(\mathrm{OD}_{600}=0.05\right.$, three technical replicates). Pe299R::mSc fluorescence was measured every 5 min for $20 \mathrm{~h}$. Fold change of Pe299R::mSc fitness relative to intraspecific competition was calculated as the binary logarithm of the ratio between the AUC of Pe299R::mSc in interspecific competition and the mean of the AUC of Pe299R::mSc in intraspecific competition.

Evaluation of the Pe299R porter was first evaluated as monoculture in liquid culture and the

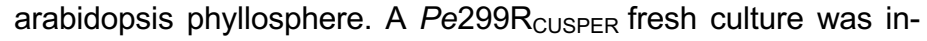
duced with $1 \mathrm{mM}$ isopropyl beta-D-1-thiogalactopyranoside (IPTG) and $50 \mu \mathrm{g} \mathrm{mL}^{-1}$ kanamycin, as described in detail in Appendix SI. The IPTG-induced culture was used to either inoculate NB or air sprayed onto arabidopsis plants. Samples were taken at different time points, in which cells were harvested, fixed, and stored at $20{ }^{\circ} \mathrm{C}$ until they were analyzed by microscopy.

Plant Inoculation. To determine the fitness effect of Pe299R CUSPER in competition with epiphytic bacteria on leaves, arabidopsis plants (Arabidopsis thaliana accession Col-0) were grown in Magenta GA-7 tissue-culture boxes containing $1 / 2 \mathrm{MS}$ agar media $(1.0 \% \mathrm{w} / \mathrm{v}, \mathrm{pH} 5.9)$. Plants were grown in a Conviron A1000 plant growth chamber at $22^{\circ} \mathrm{C}, 80 \%$ relative humidity $(\mathrm{RH})$ and short-day photoperiod (11 h light / $13 \mathrm{~h}$ dark cycles, light intensity $\sim 120-150 \mu \mathrm{E} \mathrm{m} \mathrm{m}^{-2} \mathrm{~s}^{-1}$ ). For plant inoculation, bacterial suspensions were adjusted to an $\mathrm{OD}_{600 \mathrm{~nm}}$ of 0.05 and mixed in equal ratios for each pair (1:1 competitor:Pe299R CUSPER$)$. Fourweek-old plants were inoculated with $200 \mu \mathrm{L}$ bacterial suspension per box using a sterile airbrush (KKmoon Airbrush Model T130A). Plants were harvested at 0 and 24 hpi by cutting the complete leaf rosette from the roots using sterile scissors and scalpel and transferring the plant into a $1.7 \mathrm{ml}$ microcentrifuge tube. For each condition, four independent plants were used as biological replicates. After the fresh weight of each plant was determined, $1 \mathrm{~mL}$ PBS supplemented with $0.02 \%$ Silwet L-77 was added. Samples were shaken in a bead mill homogenizer (Omni Bead Ruptor 24, Omni International) for two cycles of 5 min at a speed of $2.6 \mathrm{~m} \mathrm{~s}^{-1}$ and sonicated for 5 min (Easy $30 \mathrm{H}$, Elmasonic). Colony-forming units were determined by serial dilutions on R2A agar plates and normalized by the corresponding plant fresh weight (CFU $\mathrm{gFW}^{-1}$ ), while the remaining supernatants were transferred into a clean 1.7-mL centrifuge tube and centrifuged at $15,000 \times \mathrm{g}$ for $10 \mathrm{~min}$ at $4{ }^{\circ} \mathrm{C}$ to collect cells for microscopy. Cells were fixed in $4 \%$ paraformaldehyde in $1 \times$ PBS (Appendix SI, Materials and Methods).

Microscopy. Fixed bacterial cells recovered from leaves or from liquid culture were mounted on microscopy slides coated with $0.1 \% \mathrm{w} / \mathrm{v}$ gelatine. Images were taken on a Zeiss Axiolmager.M1 fluorescent widefield microscope at $1000 \times$ magnification (EC Plan-Neofluar $100 \times / 1.30$ Ph3 Oil M27 objective) equipped with Zeiss filter sets $38 \mathrm{HE}$ and 43HE (BP 470/40-FT 495-BP 525/50 and BP 550/25-FT 570-BP 605/70, respectively), an Axiocam 506, and the software Zeiss Zen 2.3. At least 100 cells were acquired per biological replicate in three different channels: green $(38 \mathrm{HE}$ filterset), red (43HE filterset), and phase contrast.

Image Analysis. Images were analyzed using FIJI/ImageJ (version 2.0.0-rc-69/1.52s, (42)). As Pe299R CUSPER constitutively expresses mScarlet-l, the red fluorescent channel was used as a mask to select individual cells, using the thresholding method "intermodes" and converted to a binary mask object. Only particles with a size range of $0.5-2.5 \mu \mathrm{m}$ were selected. Selected objects were manually inspected using the phase contrast images to corroborate the selection of bacterial cells and to delete false positive red fluorescent particles. The mask was then used to determine green fluorescence of Pe299R ground fluorescence was measured by sampling a random section of background area in each fluorescent image (2).

Estimation of Single-Cell Reproductive Success. The reproductive success of $P e 299 R_{\text {CUSPER }}$ at a time $t$ was estimated from background-corrected fluorescence measurements by subtracting the average fluorescence intensity of each cell by the background fluorescence of its corresponding field of view, using the 97.5 percentile point of the background intensity of each replicate (mean + $1.96 \times$ standard deviation). Then, reproductive success of a cell was calculated according to (12). The distribution of reproductive success of the initial cell population was estimated as a fraction of

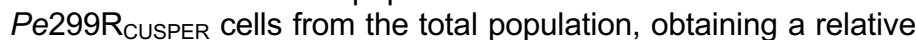
subpopulation for each condition (Appendix SI, Table S3). The similarity of the resulting distributions was compared by hierarchical clustering using the mean of the relative fraction of Pe299R CusPer $_{\text {subpopulations. }}$

\section{Acknowledgments}

We would like to thank Paula Jameson and Matthew Stott for useful discussions. This work was supported by Marsden Fast Start grant number 17-UOC-057 (M.N.P.R.-E.). R.O.S. was supported by a New Zealand International Doctoral Research Scholarship (NZIDRS) and a University of Canterbury College of Science Ph.D. scholarship. M.B. was supported by a University of Canterbury Ph.D. Scholarship.

\section{References}

1. H. K. Doan, et al., Topography-driven shape, spread, and retention of leaf surface water impacts microbial dispersion and activity in the phyllosphere. Phytobiomes J. 4, 268-280 (2020).

2. J. H. Leveau, S. E. Lindow, Appetite of an epiphyte: quantitative monitoring of bacterial sugar consumption in the phyllosphere. Proc. Natl. Acad. Sci. U. S. A. 98, 3446-3453 (2001).

3. R. Tecon, A. Ebrahimi, H. Kleyer, S. Erev Levi, D. Or, Cell-to-cell bacterial interactions promoted by drier conditions on soil surfaces. Proc. Natl. Acad. Sci. U. S. A. 115, 9791-9796 (2018).

4. A. Dal Co, S. van Vliet, D. J. Kiviet, S. Schlegel, M. Ackermann, Shortrange interactions govern the dynamics and functions of microbial communities. Nat Ecol Evol 4, 366-375 (2020).

5. M. N. P. Remus-Emsermann, R. O. Schlechter, Phyllosphere microbiology: at the interface between microbial individuals and the plant host. New Phytol. 218, 1327-1333 (2018).

6. M. N. P. Remus-Emsermann, et al., Spatial distribution analyses of natural phyllosphere-colonizing bacteria on Arabidopsis thaliana revealed by fluorescence in situ hybridization. Environ. Microbiol. 16, 2329-2340 (2014).

7. D. S. Esser, J. H. J. Leveau, K. M. Meyer, K. Wiegand, Spatial scales of interactions among bacteria and between bacteria and the leaf surface. FEMS Microbiol. Ecol. 91 (2015).

8. S. Steinberg, et al., Two-way microscale interactions between immigrant bacteria and plant leaf microbiota as revealed by live imaging. ISME J. 15, 409-420 (2021).

9. P. Chesson, Mechanisms of maintenance of species diversity. Annu. Rev. Ecol. Syst. 31, 343-366 (2000).

10. A. D. Letten, P.-J. Ke, T. Fukami, Linking modern coexistence theory and contemporary niche theory. Ecol. Monogr. 87, 161-177 (2017). 
bioRxiv preprint doi: https://doi.org/10.1101/2022.01.20.477054; this version posted January 21, 2022. The copyright holder for this preprint (which was not certified by peer review) is the author/funder, who has granted bioRxiv a license to display the preprint in perpetuity. It is made available under aCC-BY-NC-ND 4.0 International license.

Schlechter et al., 20 JAN 2022 - preprint - BioRxiv

11. M. N. P. Remus-Emsermann, J. H. J. Leveau, Linking environmental heterogeneity and reproductive success at single-cell resolution. The ISME Journal 4, 215-222 (2010).

12. M. N. P. Remus-Emsermann, R. Tecon, G. A. Kowalchuk, J. H. J. Leveau, Variation in local carrying capacity and the individual fate of bacterial colonizers in the phyllosphere. ISME J. 6, 756-765 (2012).

13. M. N. P. Remus-Emsermann, G. A. Kowalchuk, J. H. J. Leveau, Single-cell versus population-level reproductive success of bacterial immigrants to pre-colonized leaf surfaces. Environ. Microbiol. Rep. 5, 387-392 (2013).

14. D. V. Badri, G. Zolla, M. G. Bakker, D. K. Manter, J. M. Vivanco, Potential impact of soil microbiomes on the leaf metabolome and on herbivore feeding behavior. New Phytol. 198, 264-273 (2013).

15. R. C. MacDonald, R. Fall, Detection of substantial emissions of methanol from plants to the atmosphere. Atmospheric Environment. Part A. General Topics 27, 1709-1713 (1993).

16. M. Nemecek-Marshall, R. C. MacDonald, J. J. Franzen, C. L. Wojciechowski, R. Fall, Methanol Emission from Leaves (Enzymatic Detection of Gas-Phase Methanol and Relation of Methanol Fluxes to Stomatal Conductance and Leaf Development). Plant Physiol. 108, 1359-1368 (1995).

17. R. O. Schlechter, E. J. Kear, D. M. Remus, M. N. P. Remus-Emsermann, Fluorescent Protein Expression as a Proxy for Bacterial Fitness in a High-Throughput Assay. Appl. Environ. Microbiol. 87, e0098221 (2021).

18. J.-M. Monier, S. E. Lindow, Aggregates of resident bacteria facilitate survival of immigrant bacteria on leaf surfaces. Microb. Ecol. 49, 343352 (2005).

19. M. Wilson, S. S. Hirano, S. E. Lindow, Location and survival of leafassociated bacteria in relation to pathogenicity and potential for growth within the leaf. Appl. Environ. Microbiol. 65, 1435-1443 (1999).

20. R. Tecon, J. H. J. Leveau, The mechanics of bacterial cluster formation on plant leaf surfaces as revealed by bioreporter technology. Environ. Microbiol. 14, 1325-1332 (2012).

21. M. N. P. Remus-Emsermann, E. B. Kim, M. L. Marco, R. Tecon, J. H. J. Leveau, Draft Genome Sequence of the Phyllosphere Model Bacterium Pantoea agglomerans 299R. Genome Announc. 1 (2013).

22. M. Wilson, S. E. Lindow, Coexistence among Epiphytic Bacterial Populations Mediated through Nutritional Resource Partitioning. Appl. Environ. Microbiol. 60, 4468-4477 (1994).

23. A. C. Martiny, K. Treseder, G. Pusch, Phylogenetic conservatism of functional traits in microorganisms. ISME J. 7, 830-838 (2013).

24. M. Ghoul, S. Mitri, The Ecology and Evolution of Microbial Competition. Trends Microbiol. 24, 833-845 (2016).

25. N. Delmotte, et al., Community proteogenomics reveals insights into the physiology of phyllosphere bacteria.

26. J. A. Vorholt, Microbial life in the phyllosphere. Nat. Rev. Microbiol. 10, 828-840 (2012).

27. E. J. N. Helfrich, et al., Bipartite interactions, antibiotic production and biosynthetic potential of the Arabidopsis leaf microbiome. Nat Microbiol 3, 909-919 (2018).
28. J. Kehe, et al., Positive interactions are common among culturable bacteria. bioRxiv (2020) https:/doi.org/10.1101/2020.06.24.169474.

29. M. N. P. Remus-Emsermann, S. de Oliveira, L. Schreiber, J. H. J. Leveau, Quantification of lateral heterogeneity in carbohydrate permeability of isolated plant leaf cuticles. Front. Microbiol. 2, 197 (2011)

30. C. M. Vogel, D. B. Potthoff, M. Schäfer, N. Barandun, J. A. Vorholt, Protective role of the Arabidopsis leaf microbiota against a bacterial pathogen. Nature Microbiology 6, 1537-1548 (2021).

31. A. Y. Burch, V. Zeisler, K. Yokota, L. Schreiber, S. E. Lindow, The hygroscopic biosurfactant syringafactin produced by Pseudomonas syringae enhances fitness on leaf surfaces during fluctuating humidity. Environ. Microbiol. 16, 2086-2098 (2014).

32. S. Oso, M. Walters, R. O. Schlechter, M. N. P. Remus-Emsermann, Utilisation of hydrocarbons and production of surfactants by bacteria isolated from plant leaf surfaces. FEMS Microbiol. Lett. 366 (2019).

33. L. Schreiber, et al., Plant-microbe interactions: identification of epiphytic bacteria and their ability to alter leaf surface permeability. New Phytol. 166, 589-594 (2005).

34. M. Murillo-Roos, H. S. M. Abdullah, M. Debbar, N. Ueberschaar, M. T. Agler, Niche separation in cross-feeding sustains bacterial strain diversity across nutrient environments and may increase chances for survival in nutrient-limited leaf apoplasts. bioRxiv (2021) https:/doi.org/10.1101/2021.11.07.467568.

35. W. A. Corpe, S. Rheem, Ecology of the methylotrophic bacteria on living leaf surfaces. FEMS Microbiol. Lett. 62, 243-249 (1989).

36. D. B. Müller, O. T. Schubert, H. Röst, R. Aebersold, J. A. Vorholt, Systems-level Proteomics of Two Ubiquitous Leaf Commensals Reveals Complementary Adaptive Traits for Phyllosphere Colonization. Mol. Cell. Proteomics 15, 3256-3269 (2016).

37. M. Wilson, S. E. Lindow, Inoculum Density-Dependent Mortality and Colonization of the Phyllosphere by Pseudomonas syringae. Appl. Environ. Microbiol. 60, 2232-2237 (1994).

38. G. Innerebner, C. Knief, J. A. Vorholt, Protection of Arabidopsis thaliana against leaf-pathogenic Pseudomonas syringae by Sphingomonas strains in a controlled model system. Appl. Environ. Microbiol. 77, 3202-3210 (2011).

39. C. Vogel, N. Bodenhausen, W. Gruissem, J. A. Vorholt, The Arabidopsis leaf transcriptome reveals distinct but also overlapping responses to colonization by phyllosphere commensals and pathogen infection with impact on plant health. New Phytol. 212, 192-207 (2016).

40. P. Ji, M. Wilson, Assessment of the importance of similarity in carbon source utilization profiles between the biological control agent and the pathogen in biological control of bacterial speck of tomato. Appl. Environ. Microbiol. 68, 4383-4389 (2002).

41. S. S. Hirano, C. D. Upper, Bacteria in the leaf ecosystem with emphasis on Pseudomonas syringae-a pathogen, ice nucleus, and epiphyte. Microbiol. Mol. Biol. Rev. 64, 624-653 (2000).

42. J. Schindelin, et al., Fiji: an open-source platform for biological-image analysis. Nat. Methods 9, 676-682 (2012). 